\title{
TO OUR REVIEWERS: MUCHAS GRACIAS
}

Our reviewers make an invaluable contribution toward ensuring the ongoing high quality and warm reception of our journal. Their rigorous, punctual, and balanced reviews of the latest publications in the field have assisted our journal's success enormously, ensuring that we continue to be the foremost venue for scholarly conversations in the field of Latino Studies today.

As we begin our journal's sixth volume, I want to take this opportunity to express my appreciation to the reviewers of the articles we both published and considered for publication in Volume 5. I truly value your expertise and continued support for Latino Studies.

Thank you/muchas gracias!

Suzanne Oboler

Editor

Marixsa Alicea

Louis Mendoza

Paul Allatson

Isabel Molina

Héctor Avalos

Hiroshi Motomura

César Ayala

Ruth Behar

Emilio Pantojas

Elizabeth Campisi

Lisa Pasko

Loretta Capeheart

Rafael Pérez-Torres

Raphael Dalleo

Moisés Próspero

Stephen Demuth

Raymond Rocco

Jorge Duany

Ana Patricia Rodríguez

Anani Dzidzienyo

Ralph E. Rodríguez

Ronald Fernández

Rubén Rosario Rodríguez

Lisa García-Bedolla

Tom I. Romero, II

Alicia Gaspar de Alba

Jodie G. Roure

Nicole M. Guidotti-Hernández

Otto Santa Ana

Carl Gutiérrez-Jones

Laurie Schaffner

John Hagedorn

Jonathan Simon

Peter J. Spiro

Berta Esperanza Hernández-Truyol

Lucía M. Suárez

Andrea Herrera

Silvio Torres-Saillant

Kevin Johnson

Michael Jones-Correa

Daniel Kanstroom

Angharad Valdivia

Charles R. Venator Santiago

Anahi Viladrich

Iraida López

Philip J. Williams

Marta Maldonado

Diane L. Wolf

Vicki Mayer

Latino Studies (2008) 6, 243. doi:10.1057/lst.2008.21 